Conclusions Despite only a small number of patients meeting the criteria for NTM disease, each single positive NTM culture generates a cascade of further investigations and work for the mycobacterium service. As a minimum, multiple samples, radiology and symptom assessment are needed. For those NTM associated with more complex comorbid disease, this often needs to be repeated over many months.

\section{P34 5-YEAR EXPERIENCE OF LATENT TUBERCULOSIS INFECTION (LTBI) MANAGEMENT PRE- IMMUNOMODULATORY THERAPY AT A TERTIARY HOSPITAL INFECTIOUS DISEASES UNIT IN THE UK}

${ }^{1} \mathrm{~S}$ Colley, ${ }^{2} \mathrm{~N}$ Laundy, ${ }^{2} \mathrm{~A}$ Vedio, ${ }^{2} \mathrm{DB}$ Cohen, ${ }^{1} \mathrm{PJ}$ Collini. 'University of Sheffield, Sheffield, UK: ${ }^{2}$ Sheffield Teaching Hospitals NHS Foundation Trust, Sheffield, UK

\subsection{6/thorax-2021-BTSabstracts. 144}

A growing number of immunomodulatory therapies that can increase the risk of reactivating latent $\mathrm{TB}$ infection (LTBI) are being used. Accordingly a broader range of patients screened for LTBI and referred to TB clinics bring new challenges to chemoprophylaxis risk benefit assessment. We describe 5 years' experience of LTBI management in our infectious diseases TB clinic.

We collected retrospective case note data for all LTBI patients who were assessed for chemoprophylaxis in the clinic between 1/9/2015-30/9/2020. We recorded interferon gamma release assay (IGRA) and tuberculin skin test (TST) results, choice of LTBI chemoprophylaxis and completion rates, and alanine aminotransferase (ALT) levels during treatment. We compared these outcomes according to patient age and gender, referring specialty, current and planned immunomodulatory therapy.

367 patients (48\% female) were diagnosed with LTBI, 188 (51\%, 47\% female) of whom were pre-immunomodulatory therapy and significantly older (median age 56.2 vs 32.6 $\mathrm{p}<0.0001)$. This group were also more likely to have had a negative/indeterminate QuantiFERON-TB Gold (QFT) $(25.5 \%$ vs $4.1 \%, \mathrm{p}<0.0001$ ), as were the $95 / 188$ of these on immunosuppressive therapy when QFT tested $(41 \%$ vs $9 \%, p$ $<0.0001)$. In total, 25 different immunomodulatory treatments were planned by 7 referring specialties for $>20$ different conditions; anti-TNF (87 patients, 46.3\%), other biologic/small molecule inhibitors (SMI) (38, 20.2\%), checkpoint inhibitors (21, 11.2\%) and DMARDs (14, 7.4\%). Oncology referrals and the range of different agents both increased over the period, while overall numbers were stable.

90/188 (48\%) patients initiated chemoprophylaxis, 50 with isoniazid $(\mathrm{H}), 40$ with rifampicin/isoniazid (RH). Those initiating chemoprophylaxis were significantly younger (median 48.7 vs 63.5 years old, $\mathrm{p}<0.0001)$. Patients with planned anti-TNF $(49,56 \%)$ were more likely to initiate chemoprophylaxis than for other biologic/SMI $(15,39 \%)$ or immune checkpoint inhibitor therapy $(19,6 \%) .16(18 \%)$ patients had any rise in ALT, $5(6 \%)>5$ times the upper limit of normal. $67(74 \%)$ completed chemoprophylaxis, 6 stopped early due to ALT rises. No differences in these outcomes were seen according to chemoprophylaxis agent, gender or age.

Fewer than half pre-immunotherapy LTBI patients commenced chemoprophylaxis, reflecting older age, QFT uncertainty and an increasing variety of biologics/SMIs.

\section{P35 NECK NODE TB: PERSPECTIVE FROM THE RAPID ACCESS HEAD AND NECK CLINIC}

R Noonan, M Walker, S Burns, D Rana, N Mani, SO Brij. Manchester Royal Infirmary, Manchester, UK

\subsection{6/thorax-2021-BTSabstracts. 145}

Background Lymph node TB is the commonest form of extra pulmonary TB and in particular, neck node TB (approximately $20 \%$ local data). The 'neck lump pathway' was interrogated to understand differential diagnosis with regards $\mathrm{TB}$ and other pathologies.

Aim To assess fine needle aspiration cytology (FNAC) in the Rapid Access Head and Neck Clinic (RAHNC) to facilitate patient assessment, next investigation and appropriate timely out-patient review.

Method A retrospective review of all referrals during the period 01/01/2019-01/12/2019 was undertaken to assess whether initial assessment with FNAC was sufficient to plan on-going management of active TB.

Results 203 neck lump referrals were reviewed in RAHNC (median time from referral 9 days, IQR 4-35) as their first 2 week wait appointment; median age 53 (IQR 35-65); male gender 111 (55\%); non-Caucasian ethnicity 83 (41\%).

All referrals had initial FNAC and 18 (9\%) underwent a second (table 1). First FNAC was diagnostic in $76 \%$ of malignant neck lumps. Further histology for diagnosis of cancer and/or staging required in $28(44 \%)$ and in all lymphoma patients. A significant number of benign conditions (27\%) required surgical biopsy to exclude malignancy.

$\mathrm{TB}$ suspected in $17(8 \%)$; median age 42 (IQR 31-51); non-Caucasian ethnicity 13 (76\%); initial FNA consistent with TB cytology (7 granulomatous; 8 necrotising; 1 caseating inflammation; 1 pus). A single patient underwent a second FNA for TB culture. 5 (29\%) required excision biopsy (3 confirmed TB; 2 lymphoma).

TB diagnosed in $14(7 \%)$ with 64\% culture positivity: 7 fully sensitive; 1 Isoniazid resistance; 1 Rifampicin monoresistance; 3 culture negative; 2 culture not performed).

Median time from first attendance to TB treatment start 47 days (IQR 27-76). All persons started on TB therapy completed their intended regimen duration.

Conclusion The RAHNC effectively investigates neck lumps of varied pathologies. A single FNA may be sufficient to

Abstract P35 Table 1 Fine needle aspiration cytology (FNAC) result by diagnosis and requirement for further histological clarification

\begin{tabular}{|c|c|c|c|c|c|}
\hline \multirow[b]{2}{*}{$\begin{array}{l}\text { Head and } \\
\text { neck cancer }\end{array}$} & \multirow{2}{*}{$\begin{array}{l}\text { Final } \\
\text { diagnosis } \\
\text { frequency } \\
(\%) \\
45(22)\end{array}$} & \multicolumn{2}{|c|}{$\begin{array}{l}\text { 1st FNAC } \\
\text { diagnostic (\%) }\end{array}$} & \multirow{2}{*}{$\begin{array}{l}\begin{array}{l}\text { Further } \\
\text { histological } \\
\text { diagnosis } \\
\text { required }\end{array} \\
8\end{array}$} & \multirow{2}{*}{$\begin{array}{l}\text { Histology } \\
\text { required for } \\
\text { IHC/staging } \\
20\end{array}$} \\
\hline & & $61(76)$ & $37(82)$ & & \\
\hline $\begin{array}{l}\text { Metastatic } \\
\text { cancer }\end{array}$ & $16(8)$ & & $12(75)$ & 4 & 2 \\
\hline Lymphoma & $19(9)$ & & $12(63)$ & 7 & $19(100 \%)$ \\
\hline TB & $14(7)$ & $11(79)$ & & 3 & NA \\
\hline Benign other & $109(53)$ & $73(67)$ & & $29(27 \%)$ & NA \\
\hline
\end{tabular}


diagnose TB (79\%). However, TB cytology is not specific and in particular, lymphoma cannot be excluded. However, despite early intervention with FNAC, there remains a relatively long duration before TB treatment is commenced.

\section{P36 SCREENING FOR BLOOD-BORNE VIRUSES AND VITAMIN D DEFICIENCY IN PATIENTS WITH ACTIVE AND LATENT MYCOBACTERIUM TUBERCULOSIS (TB) INFECTION IN THE UK: A LONGITUDINAL COHORT STUDY}

HM Chen, F Rabbani, N Clerk, K Young, E Lunn, S Kalam, G Antunes. The James Cook University Hospital, Middlesbrough, UK

\subsection{6/thorax-2021-BTSabstracts. 146}

Introduction Routine Human Immunodeficiency Virus (HIV) and viral hepatitis screening for newly diagnosed active and latent TB infection (LTBI) respectively are recommended by NICE, but there is sparse data relating to screening for Vitamin D (Vit D) deficiency in both active and LTBI infection in the UK however.

Aims The assessment of the screening rates and prevalence of HIV, viral hepatitis (B and C) and Vit D deficiency in adults with newly diagnosed active and LTBI.

Methods A longitudinal prospective study conducted over a five year period in a tertiary hospital in the North East of England. Data were obtained from a specific service database and examined ethnicity; blood-borne viral status; Vit D levels; liver and kidney function at diagnosis.

Results A total of 259 patients were diagnosed with TB during the study period (92 active; 167 latent). The main ethnic groups were Black African (34\%), Caucasian (32\%), and Indian Subcontinent (19\%).

HIV testing was performed in 87 (95\%) of patients with active TB, while 141 (84\%) were tested in the LTBI group. Four patients and a single individual were newly diagnosed with HIV infection in the active and LTBI groups, respectively.

Viral Hepatitis serology was performed in $62 \%$ and $82 \%$ of patients with active and LTBI respectively. Seven patients were newly diagnosed with Hepatitis B infection in the LTBI group.

Vit D levels were performed at baseline in 107 (41\%) patients with a median level of $33 \mathrm{nmol} / \mathrm{L} .37$ (35\%) individuals had levels below $25 \mathrm{nmol} / \mathrm{L}$. Vit D levels were significantly lower in the active TB group $(\mathrm{p}=0.0004)$.

Chronic liver and renal disease were diagnosed pre-treatment in 5\% and 2\% respectively.

Conclusions Screening for HIV and viral Hepatitis infection remained high for both groups during this period with a small but significant number of new diagnoses. Although Vit D deficiency remains contentious, the study provides evidence to support routine screening but larger longitudinal studies where cost-effectiveness is assessed are warranted.

\section{P37 MANAGING NON TUBERCULOUS MYCOBACTERIAL PULMONARY DISEASE - MISSED OPPORTUNITIES?}

Y Maung Maung Myint, A Jacob, S Ananth, C Stavropoulos, A Hawkins, M Vidwans, P Nandasiri, A Jayaratnam. West Hertfordshire Hospitals NHS Trust, Watford, UK
Introduction Non-tuberculous mycobacterium pulmonary disease (NTM-PD) can be a cause of significant pulmonary disease. Early diagnosis and treatment is important. In this review we studied the investigation, treatment and outcomes of patients who had microbiological confirmation of a nontuberculous mycobacterium (NTM).

Methods A review of all patients from 2017 - 2019 with a single sputum or bronchial wash culture of NTM was undertaken with notes, microbiology and radiology review. Further analysis was done to review whether patients were managed according to the BTS guidelines. ${ }^{1}$

Results Data for 135 patients was analysed, epidemiological and clinico-radiological findings are shown in table 1. 30 patients were treated for NTM-PD. 21 met the criteria for treatment.

$17 / 30$ had repeat sputum cultures at the end of the treatment. 13/30 achieved showed sputum conversion. 12/30 of treated patients showed improved outcomes at treatment completion. 2 were unchanged; 4 died (unrelated to NTM). The remainder, 12/30, remain under follow up.

$105 / 135$ of patients with single sputum isolates for AAFB were not treated. 32/105 had evidence of NTM-PD. Reasons for not treating are as follows - 11/32 had mild symptoms only, $8 / 32$ had other significant co-morbidities causing symptoms, 3/32 refused treatment, 3/32 reason unclear, 3/32 not

Abstract P37 Table 1 Clinical parameters of the patients with positive NTM culture

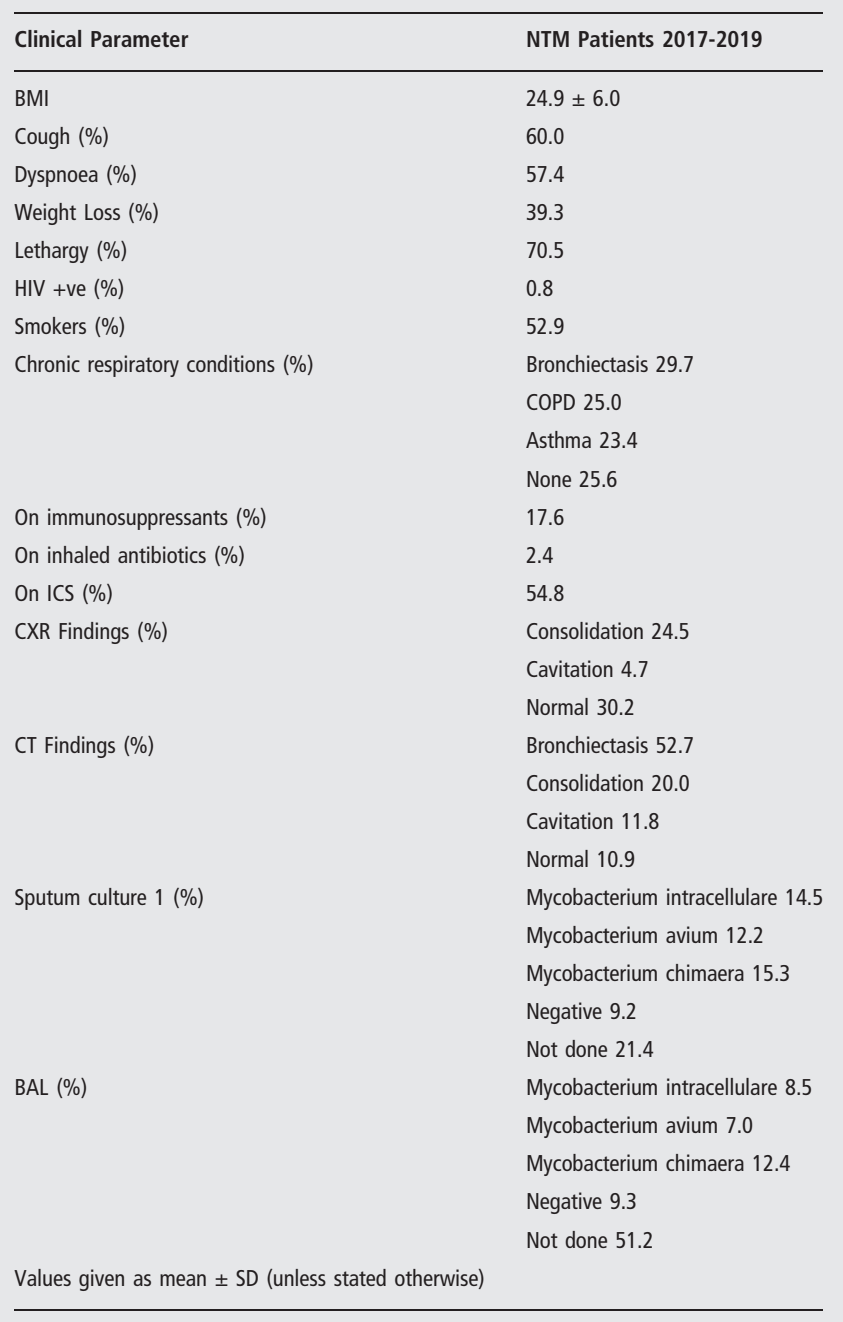

